\title{
New Therapeutic Approaches in Pulmonary Embolism
}

\author{
Sebastian M. Schellong Benjamin A. Schmidt \\ Division of Angiology, University Hospital Carl Gustav Carus, Technical University Dresden, Dresden, Germany
}

\section{Key Words}

Venous thromboembolism - Thrombolysis - Coagulation

\begin{abstract}
Pulmonary embolism as a part of venous thromboembolic disease has a broad spectrum of clinical presentations from minimal disease to life-threatening right heart failure. Therapy has to be guided by the risk associated with the individual clinical state of the patient. As long as hemodynamics are entirely stable, anticoagulation is given in order to prevent early or late recurrence, thereby allowing for endogeneous thrombolysis and recovery. In hemodynamically instable patients, i.e. patients under cardiopulmonary resuscitation or in shock, there is the
\end{abstract}

Previous articles in this series: $\quad 1$. Kroegel C, Reissig A: Principle mechanisms underlying venous thromboembolism: Epidemiology, risk factors, pathophysiology and pathogenesis. Respiration 2003;70:7-30. 2. Meyer G, Roy PM, Sors H, Sanchez O: Laboratory tests in the diagnosis of pulmonary embolism. Respiration 2003;70:125-132. 3. Garg K, Macey L: Helical CT scanning in the diagnosis of pulmonary embolism. Respiration 2003;70:231237. 4. Schümichen C: V/Q-Scanning/SPECT for the diagnosis of pulmonary embolism. Respiration 2003;70:329-342. 5. Reissig A Kroegel C: Transthoracic ultrasound of lung and pleura in the diagnosis of pulmonary embolism. A novel non-invasive bedside approach. Respiration 2003;70:441-452.

\section{KARGER}

Fax +4161306 1234

E-Mail karger@karger.ch

www.karger.com
(C) 2003 S. Karger AG, Basel

0025-7931/03/0706-0559\$19.50/0

Accessible online at: www. karger.com/res need for a rapid reduction of thrombus mass in order to restore right ventricular function. Systemic thrombolysis is the most feasible modality to reduce the thrombus burden of the pulmonary circulation in the short term. For hemodynamically stable patients with right ventricular dysfunction as assessed by echocardiography, there is still some controversy as to whether thrombolysis improves the long-term outcome. At the least, thrombolysis may positively modify the short-term course of acute disease in patients with an extremely low risk of bleeding. When the acute phase has been overcome, secondary prophylaxis with vitamin $\mathrm{K}$ antagonists has to be given. The duration of secondary prophylaxis requires an individual assessment of both the risk of recurrence and the risk of bleeding. In the near future, new anticoagulant drugs such as direct thrombin and factor Xa inhibitors will offer new treatment modalities for the acute phase as well as for secondary prophylaxis.

Copyright $@ 2003$ S. Karger AG, Basel

\section{Introduction}

Pulmonary embolism (PE) may be considered a manifestation of a more complex disease, i.e. venous thromboembolic disease. Two basic facts justify this concept. Firstly, in more than $90 \%$ of patients with PE, the origin

Sebastian M. Schellong, Division of Angiology

University Hospital Carl Gustav Carus, Technical University Dresden

Fetscherstrasse 74, DE-01277 Dresden (Germany)

Tel. +49 351458 3659, Fax +49 3514584359

E-Mail sebastian.schellong@mailbox.tu-dresden.de 
of the thrombus can be found as deep venous thrombosis (DVT) of the leg veins. Secondly, in more than $50 \%$ of all DVT patients without signs and symptoms of PE, perfusion defects are present on a lung scan. Considering venous thromboembolic disease as a unifying concept, it is plausible that treatment of this disease should be to some extent uniform. Indeed, most of the data regarding therapy of PE are shared with data referring to DVT. In some aspects, treatment recommendations for PE are merely extrapolated from evidence established for DVT, since those aspects have not been studied separately for PE.

On the other hand, PE may be very different from DVT. This is particularly true if PE leads to hemodynamic sequelae by stressing the right heart on a broad spectrum of degrees of severity from mild increase of right ventricular afterload to frank right heart failure. Therapeutic measures in this situation are directed towards decreasing right ventricular afterload, which may be accomplished by decreasing thrombus mass in the pulmonary arteries. The following paragraphs review all modalities for the treatment of PE. Finally, an integrated concept covering all clinical presentations of $\mathrm{PE}$ will be discussed.

\section{Initial Anticoagulation}

In 1960, Barritt and Jordan [1] published a randomized trial about the treatment of PE. Remarkably enough, they randomized patients with acute PE into a treatment arm, consisting of anticoagulation with heparin followed by a 2-week course of a vitamin $\mathrm{K}$ antagonist (VKA), or no treatment at all [1]. In those days, at a time without established pharmacological thromboprophylaxis, the fear of refractory bleeding induced by the use of anticoagulants early after an operation was much greater than the fear of even life-threatening venous thromboembolism (VTE). For that reason, no ethical objections were made against this otherwise methodologically sound trial. It was probably due to the same reason that the authors expressed surprise when they found they had to stop the trial after randomizing 35 patients, of whom 19 had received no treatment and 16 had been treated with anticoagulants. Five patients without treatment had died from recurrent PE, and another 5 had suffered nonfatal recurrence, while among the patients on anticoagulants, no fatal or nonfatal PE had occurred. After the first 35 patients, randomization was stopped and the treatment arm of the study continued to a total of 54 patients; only one nonfatal recurrence of PE and one episode of fatal bleeding associated with unduly long prolongation of prothrombin time were reported.

Even without sample size or power calculation, this trial provided evidence strong enough to establish anticoagulation as the routine treatment in patients with confirmed PE. This treatment was extrapolated to benefit patients with DVT as well, and no further trials on this topic were ever conducted.

Over the next 25 years, trials on anticoagulation did not specifically refer to patients with PE but were conducted within a more comprehensive concept which considered DVT and PE as different manifestations of the same disease. The classic sequence of unfractionated heparin (UFH) followed by VKAs was refined in several aspects: (1) UFH dosage should be guided by monitoring activated partial thromboplastin time (aPTT), with a prolongation of 1.5-2.0 times the upper limit of normal being appropriate [2]; (2) subcutaneous instead of intravenous administration of UFH is adequate provided that aPTT is monitored according to an appropriate time schedule [3]; (3) dose adjustments of UFH should ideally follow a predefined decision rule, so-called nomograms, indicating the appropriate change of dosage in response to aPTT deviation from the target range - proposals for such nomograms including validation trials have been made until very recently $[4,5]$; (4) UFH administration does not need to be extended beyond 10 or more days, and 5 days are generally sufficient [6], and (5) even if VKAs are started on the day of diagnosis, the concurrent administration of heparin for the first 5 days is necessary to effectively treat VTE [7].

These evidence-based refinements of initial anticoagulation therapy had certainly not completely penetrated current practice in the Western world when, in the early 1990s, the development of low-molecular-weight heparins (LMWHs) moved from thromboprophylaxis to therapeutic indications. For regulatory reasons, LMWHs were tested first in patients presenting with DVT. It was not until 1997 that two trials also included patients presenting with PE.

The COLUMBUS trial included 1,021 patients covering the full spectrum of VTE from isolated calf vein thrombosis to symptomatic PE, randomizing them either to classical anticoagulation with UFH or the LMWH reviparin, followed by a VKA [8]. $27 \%$ of all patients had presented with signs or symptoms of PE. The overall result of the trial was within the range of all comparable studies testing LMWHs against UFH and found no significant difference regarding the endpoints of recurrence, major 
bleed and death after 90 days of follow-up. Regarding the subgroup of patients with PE, the result was exactly the same as for the entire patient population. However, the sample size of this subgroup was not sufficiently powered to draw firm conclusions on patients presenting with PE.

At exactly the same point in time, the Tinzaparine ou Heparine Standard: Evaluations dans l'Embolie Pulmonaire study was published [9]. A comparison between LWMH and UFH, this trial exclusively enrolled patients presenting with signs and symptoms of PE. Of 612 patients, $47 \%$ had vascular obstruction of the pulmonary circulation of more than $50 \%$, mainly calculated by qualifying perfusion lung scans at study entry. However, patients in an unstable condition with overt or imminent circulatory or respiratory failure were excluded. Patients were randomized between body weight-adjusted subcutaneous LMWH (tinzaparin) once daily and intravenous UFH; both regimens were followed by treatment with a VKA for 90 days. The event rates for recurrence, major bleed and death were unexpectedly low and showed no significant differences between the LMWH and UFH. However, the study was not sufficiently powered for a formal equivalence analysis.

No trials testing any other LWMH for the treatment of PE have been performed, and most probably never will be. However, in subsequent years, the data from those two studies helped establish the growing evidence which showed the relative efficacy and safety of LMWHs in the initial treatment of VTE. A recent meta-analysis of 14 randomized controlled trials comparing LMWHs to UFH concluded that LMWHs are superior to UFH regarding the endpoint of major bleeding [10]. A strong trend towards superiority - albeit not statistically significant was also found regarding recurrent thromboembolic events. The superiority of LMWHs found with respect to mortality was achieved exclusively in a subgroup of patients with cancer and VTE. This observation gave rise to new study hypotheses about possible tumor-modulating effects of LMWHs. Thus, the current understanding of initial anticoagulation of PE can be summarized as follows: As PE is one manifestation of venous thromboembolic disease, the evidence from all methodologically sound LMWH trials applies to this particular manifestation as well as to the entire spectrum of the disease. This indicates that LMWHs in therapeutic doses are at least as safe and effective as UFH in the initial anticoagulation of $\mathrm{PE}$ and should be considered the current standard of therapy. This holds true in particular in the light of the pharmacokinetic properties of LMWHs, mainly their high bioavailability and longer half-life, which allow once or twice
Table 1. Body weight-adjusted dosage of LMWHs in the therapy of VTE

\begin{tabular}{|c|c|}
\hline LMWH & Dosage \\
\hline Certoparin ${ }^{1}$ & 8,000 IE bid (Mono-Embolex ${ }^{\circledR}$ ) \\
\hline Dalteparin $^{1}$ & $\begin{array}{l}100 \mathrm{IE} / \mathrm{kg} \text { bw bid }\left(\text { Fragmin }{ }^{\circledR}\right) \\
200 \mathrm{IE} / \mathrm{kg} \text { bw od }\left(\text { Fragmin }^{\circledR}\right)\end{array}$ \\
\hline Enoxaparin ${ }^{1}$ & $1 \mathrm{mg} / \mathrm{kg}$ bw bid $\left(\right.$ Clexane $\left.^{\circledR}\right)$ \\
\hline Nadroparin $^{1}$ & $\begin{array}{l}0.1 \mathrm{ml} / 10 \mathrm{~kg} \text { bw bid (Fraxiparin }{ }^{\circledR} \text { ) } \\
0.1 \mathrm{ml} / 10 \mathrm{~kg} \text { bw od (Fraxodi }{ }^{\circledR} \text { ) }\end{array}$ \\
\hline Reviparin $^{1}$ & $87.5 \mathrm{IE} / \mathrm{kg}$ bw bid $\left(\right.$ Clivarin $\left.{ }^{\circledR}\right)$ \\
\hline Tinzaparin & 175 IE/kg bw od (Innohep $\left.{ }^{\circledR}\right)$ \\
\hline
\end{tabular}

Observed drug information regarding body weight classes and maximal dosage. $\mathrm{bw}=$ Body weight; od = once daily; bid = twice daily.

1 Not explicitly approved for treatment of PE.

daily subcutaneous administration without laboratory monitoring and dose adjustment (fig. 1).

Two major limitations have to be borne in mind. Firstly, LMWHs have been tested only in patients without severe impairment of renal function. Elimination of LMWHs is primarily renal, so the possibility of accumulation and overdosing has to be considered in patients with impaired renal function. There is no established dose adjustment regimen for different degrees of renal impairment for any of the LMWHs. Thus, patients with a serum creatinine of $>180 \mu \mathrm{mol} / \mathrm{l}(2.0 \mathrm{mg} / \mathrm{dl})$ should be treated with UFH and the traditional aPTT-guided dose adjustment instead of an LMWH. The second limitation refers to the clinical presentation of PE. All PE patients included in clinical trials with LMWHs were, as a result of the exclusion criteria, in a stable condition without any need for catecholamines, mechanical ventilation or cardiopulmonary resuscitation (CPR). For this reason, the use of LMWHs for initial anticoagulation of PE should be considered to be safe and effective only in clinically stable patients. Another noteworthy aspect is that formal authority approval of LMWHs in PE is restricted to tinzaparin, which reflects the international trial status (table 1).

Recently, new anticoagulants have been developed with more specific characteristics in the inhibition of the coagulation cascade. These drugs exclusively target either activated factor II or activated factor X. Clinical investigation has advanced most for ximelagatran/melagatran, which is an orally administered direct thrombin inhibitor, and for fondaparinux, a parenterally administered antithrombin-dependent factor Xa inhibitor [11-13]. 
Fig. 1. Coagulation cascade and interaction of antithrombotic drugs. $F=$ Factor.

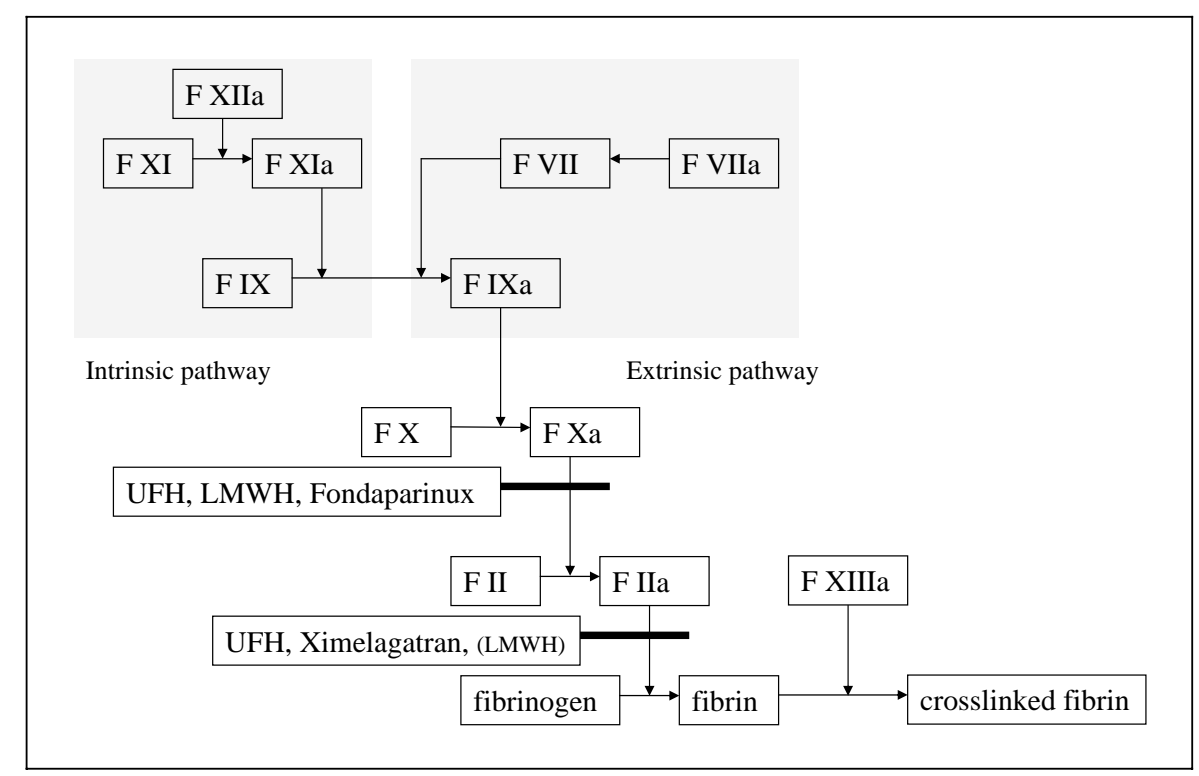

The clinical trial program of ximelagatran/melagatran does not comprise studies specifically enrolling patients with symptomatic PE. Thus, to date there are no data regarding its efficacy and safety in the initial treatment of PE. Fondaparinux has been investigated in large trials evaluating its efficacy and safety in the initial anticoagulation of patients with DVT and of patients with symptomatic PE. The trial which investigated treatment of PE (MATISSE-PE) [13] included more than 2,200 patients, who were randomized to a body weight-adjusted dose of fondaparinux ( $7.5 \mathrm{mg}$ for most patients) or UFH. Both regimens of initial treatment were followed by VKAs. The choice of UFH in the control group reflects the international standard of care at the time the trial program was designed (1998/1999).

As it was designed as a noninferiority trial, a safety margin of $+3.5 \%$ difference in the efficacy endpoint was set to define the range of noninferiority. The rate of VTE recurrence within 90 days was 3.8\% for fondaparinux and $5.0 \%$ for UFH, respectively. The $1.2 \%$ absolute risk reduction had a $95 \%$ confidence interval ranging from $-3.0 \%$ to $+0.5 \%$, thereby lacking statistically significant superiority. No significant differences were detected regarding major and minor bleeding and mortality. These results show that a once daily subcutaneous dose of fondaparinux is at least as safe and effective as UFH in the initial anticoagulation of PE. It has to be noted that fondaparinux has been studied neither in hemodynamically unstable patients nor in patients with impaired renal function. Because of the pharmacological profile of this entirely synthetic drug with no remaining immunogenicity for HIT-II antibodies and its animal-independent production, it may be anticipated that fondaparinux will gain major importance in the treatment of PE. Approval for this indication is expected in 2004.

\section{Secondary Prophylaxis}

VKAs are the current standard of secondary prophylaxis in VTE. Any specifications for treatment, such as intensity and duration, have been developed from studies with patients who for the most part had DVT as the presenting event of VTE. By the end of the 1980s, two important issues regarding secondary prophylaxis had been settled. Firstly, laboratory monitoring of anticoagulation with VKA should be done using the international normalized ratio (INR) rather than prothrombin time (Quick test). INR corrects for the substantial differences in prothrombin time that occur between thromboplastin preparations used in different tests. Although this fact has been established beyond doubt, the exclusive use of INR to monitor VKA has so far not become routine practice in many countries and continues to be an issue in medical education. Secondly, after recognition of the issue of anticoagulation intensity ranges, the optimal INR range for secondary prophylaxis of venous thromboembolic disease is 2.0 3.0. A value above 3.0 increases bleeding without improv- 
ing efficacy, while a value below 2.0 is less effective without reducing the risk of bleeding $[14,15]$. According to a recent randomized trial, the moderate range of 2.0-3.0 appears to be sufficient even for patients with VTE and antiphospholipid syndrome [16], in whom an INR higher than 3.0 had previously been suggested to be the most efficient prophylaxis [17]. Considering these data, it is now settled that whenever VKAs are used in the secondary prophylaxis of VTE, the optimal INR range is 2.0-3.0.

The issue of duration of secondary prophylaxis has been studied extensively. Although it cannot be reviewed in detail here, the following principles can be outlined:

(1) The risk of recurrent PE is very low during treatment with VKAs [18].

(2) If secondary prophylaxis is discontinued too early after the index event, an overshoot of relapses has been observed [19].

(3) If VKAs are discontinued 3 or 6 months after the initial event, recurrence will occur with a constant overall frequency of about $5 \%$ per year [19]. The individual risk of recurrence depends on a combination of transient and persistent risk factors and may be higher in an individual patient. It appears to be appropriate to prolong secondary prophylaxis for subgroups of patients with an increased risk of recurrence [20].

(4) VKA treatment of patients with VTE with an INR target range of 2.0-3.0 will cause major bleeds with a constant overall frequency of about $3 \%$ per year $[15,21]$. The individual risk of major bleeding may be higher and depends on a combination of patient characteristics. Prediction models have been developed for the risk estimation in subgroups of patients [22, 23].

There are subgroups of patients for whom randomized trials proved prolongation of secondary prophylaxis with VKAs to be beneficial. In a study of secondary prophylaxis after recurrent VTE, Schulman et al. [24] demonstrated a benefit for patients allocated to indefinite treatment after a median follow-up of 4 years. However, the study data suggested that prolongation beyond 48 months might lead to inversion of the risk-benefit ratio due to accumulating bleeding episodes [24].

An increased risk of recurrence has also been observed in patients with VTE not triggered by transient risk factors or underlying disease, such as trauma, immobilization, recent surgery, hormone therapy, pregnancy or cancer. Such episodes are termed idiopathic thromboembolism. In 1999, Kearon et al. [25] showed that after a first idiopathic episode of VTE, prolongation of VKA treatment for up to 14 months yields a positive risk-benefit ratio when compared to 3 months of treatment. However,
2 years later, Agnelli et al. [26] presented a randomized controlled follow-up study with the same approach indicating that the group with prolonged secondary prophylaxis experienced a rebound in recurrent events after discontinuation of medication, which annihilated the benefit achieved in the first year of treatment. No data have been published to reconcile these findings. However, there is a broad consensus that prolonged secondary prophylaxis after a first event of VTE should only be considered in patients with an unprovoked episode.

In order to offer prolonged secondary prophylaxis to patients with idiopathic VTE without increasing the risk of bleeding, a low-intensity regimen of VKAs (INR 1.52.0) has been tested. Unfortunately, two prospective trials revealed conflicting results. The comparison of a VKA at an INR of 1.5-2.0 with placebo showed effectiveness of this treatment regimen; firm conclusions regarding the bleeding risk could not be drawn due to a lack of statistical power for this endpoint [27]. The comparison of a VKA at an INR of 1.5-2.0 with the traditional intensity of INR 2.0-3.0 revealed a lower efficacy of the former with an equal bleeding risk, thereby indicating that the low-intensity regimen is not superior [15]. Comparing the event rates in the low-intensity arm of both trials suggests that slightly different patient populations were studied. In summary, the issue of low-intensity VKA regimens has not been settled yet.

Thrombophilia has been suggested to be a major determinant of the risk of recurrence of VTE [28]. However, after more than 10 years of clinical research, it has become clear that a thrombophilic state as assessed by laboratory testing, even though established as a risk factor for VTE, does not predict a significantly higher risk of recurrence [29]. Except for the antiphospholipid syndrome, severe antithrombin deficiency and severe protein $\mathrm{C}$ deficiency with a positive family history, prolonged secondary prophylaxis is not indicated in patients after a first VTE episode on the basis of a positive thrombophilia test result alone.

A certain number of patients with VTE are not eligible for treatment with VKAs. This may be due to low compliance, uncontrolled addictive disease or severe comorbidity. The approach of using LMWHs for secondary prophylaxis has been tested for different LMWHs in various dose regimens ranging from a high-risk prophylactic dose to a full therapeutic dose. A meta-analysis of these mostly small trials demonstrated that compared with VKAs, LMWHs are equally effective and may reduce the number of bleeding events significantly (up to 60\%) [30]. Feasibility and reimbursement arguments may prevent this ap- 
proach from becoming widespread clinical practice, but in selected patients ineligible for VKA, administration of an LMWH is an alternative option. With no consistent evidence-based recommendation available, an LMWH dosage of 80-100 IU anti-Xa per kilogram of body weight once daily appears a reasonable regimen once initial treatment has been completed.

It has to be noted that many of the data discussed above have been acquired in patients with DVT as the presenting episode of VTE. Symptomatic PE was present only in (a minor) part of the study populations. There are no trials specifically addressing the secondary prophylaxis of PE. This may prove to be an important aspect for future developments because evidence has emerged that the natural history of recurrent disease after PE is not the same as that after DVT [18]. Ninety percent of patients relapse with the clinical presentation of their first event. That means that $90 \%$ of recurrences after initial PE episodes will again be symptomatic PE. In recurrent episodes, a higher mortality has also been observed for PE as compared with DVT [18]. However, although continuous anticoagulation prevents recurrence, a rebound after discontinuation results in equal long-term recurrence rates when extended and short-term duration of VKA is compared [31]. Further evaluation is needed here since so far the appropriate response to these observations remains unclear.

Major impact on secondary prophylaxis is expected from new drug developments in the near future. Ximelagatran/melagatran is an orally administered direct thrombin inhibitor which was tested in a fixed twice daily dose over 18 months (THRIVE III study) [12]. After the end of at least 6 months of secondary prophylaxis, patients with VTE were randomized to ximelagatran/melagatran or placebo. There was a relative risk reduction of $83 \%(2 \mathrm{vs}$. $12 \%)$ for recurrent VTE events. The incidence of major bleeding was equal to that in the placebo group. Even if this result was established in patients with DVT as the primary event, there is no reason for not applying it to prolonged secondary prophylaxis after PE. However, ximelagatran/melagatran causes transient liver enzyme elevations in $5-10 \%$ of patients $[11,12]$. The nature and clinical significance of this phenomenon are not yet fully understood.

Another pharmacological principle is currently under clinical evaluation in phase III trials. Modification of the pentasaccharide molecule of fondaparinux led to a new compound (idraparinux) with a plasma half-life increased by a factor of more than 3 [32]. This allows stable anticoagulation with once weekly subcutaneous injections. Two clinical trials on the treatment of VTE with idraparinux are currently under way, enrolling 2,200 patients with DVT and another 2,200 patients with PE. Prolongation of secondary prophylaxis will also be evaluated.

\section{Systemic Thrombolysis}

Systemic thrombolysis in appropriate dosages is able to rapidly reduce thrombus mass throughout the circulation. In PE, this has been demonstrated by reduction of the Miller score, improvement of perfusion scan, reduction of pulmonary artery pressure and improvement of right ventricular function within $24 \mathrm{~h}$ after medication [33].

It is obvious that these early effects can be beneficial in patients with acute life-threatening PE, whose short-term prognosis is determined by the degree of right ventricular failure due to increased pulmonary artery pressure caused by obstruction of the pulmonary artery tree by embolic material. Acute life-threatening PE is understood as circulatory arrest and the need for CPR, or formal shock (systolic arterial pressure $<100 \mathrm{~mm} \mathrm{Hg}$, heart rate $>100 / \mathrm{min}$ ) with or without the need for ventilatory support. From a number of cohort studies, it can be estimated that mortality in these patients reaches around $70 \%$ when thrombolysis is withheld, which outnumbers by far any risk of severe bleeding even in early postoperative patients [34]. Obviously, this group of patients is not suited for randomized controlled trials, and thrombolytic therapy is considered the treatment of choice $[35,36]$.

Today, the most common dose regimen is $100 \mathrm{mg}$ of alteplase administered over $2 \mathrm{~h}$ with a front load of 10 or $20 \mathrm{mg}$. Alternatively, 2 million units of urokinase can be given over 2-4 h. Under CPR, bolus administration of 2 $\times 50 \mathrm{mg}$ or $1 \times 100 \mathrm{mg}$ of alteplase may be considered in order to achieve immediate effects. In postoperative patients and in patients undergoing CPR, major bleeding has to be expected and may require vigorous treatment with transfusion of packed red blood cells, fresh frozen plasma and platelets. There are some data that show that bleeding complications can be controlled more successfully than deterioration of right heart failure. As a clinical decision rule, CPR should not be stopped until (1) the maximum dose of a thrombolytic agent has been administered, and (2) sufficient time has elapsed to allow for effective thrombolysis and right heart recovery. Bedside transthoracic echocardiography is a helpful tool in guiding therapy $[37,38]$. 
Whether the early hemodynamic benefit of systemic thrombolysis translates into a decrease in mortality in patients with a wider scope of PE symptoms has been under investigation since the 1970s. Alteplase as well as streptokinase and urokinase have been evaluated in different dose and time regimens [33]. Control groups were treated with UFH in therapeutic dosages. Follow-up for mortality was 12 months in some trials, but unfortunately, the more recent alteplase trials only reported 30-day results. A meta-analysis performed by Dalen et al. [33] in 1997 showed no reduction of mortality with systemic thrombolysis, neither at 30 days nor at 12 months, but found an average incidence of intracranial hematoma of $2 \%$, which is higher than in thrombolysis trials of myocardial infarction. Thus, it became evident that a better definition was needed of the target population beyond those in shock or under CPR. This required a predictive measure for increased mortality in patients with PE.

Two large registries independently established that right ventricular dysfunction as assessed by transthoracic echocardiography provides such a measure. In the International Cooperative Pulmonary Embolism Registry, right ventricular dysfunction was an independent predictor of death with a hazard ratio of 2.0 [39]. The MAPPET registry showed that right ventricular dysfunction at presentation was present in $84 \%$ of patients who died, and in $16 \%$ of those who survived [34]. In both registries, right ventricular dysfunction was defined by contractility patterns of the right heart (RVESP $>30 \mathrm{~mm}$, paradoxical septum movement, right ventricular akinesia or dyskinesia) rather than by pulmonary artery pressure. PE patients with these criteria appeared most likely to benefit from thrombolysis.

Konstantinides et al. [40] conducted a randomized controlled trial from 1997 to 2001, enrolling 256 patients with submassive PE at 49 study sites. Hemodynamically stable patients with significant right ventricular dysfunction were randomized to receive either $100 \mathrm{mg}$ of alteplase over $2 \mathrm{~h}$ or placebo. The primary endpoint was a composite of death or treatment escalation. Treatment escalation was defined by infusion of catecholamines, secondary open-label thrombolysis, endotrachel intubation, CPR or mechanical thrombus fragmentation. Patients were followed until hospital discharge. For this composite endpoint, a statistically significant risk reduction of 55\% for alteplase was found. The incidence of major bleeding was extraordinarily low $(2.3 \%)$, with no cases of intracranial hemorrhage.

The interpretation of this study remains controversial. The authors claim that this study proves the benefit of systemic thrombolysis in well-defined patients and that thrombolysis should therefore be considered routinely as an option in those patients [40]. Opponents argue that the difference in outcome was exclusively due to the frequency of secondary thrombolysis within the first 4 days and to a treatment escalation which had no objective measure but was left entirely to the discretion of the attending physician. This is of particular importance since clinical observation de facto unblinded treatment assignment a few hours after administration of the study drug. There was no difference in mortality or documented recurrent PE. In addition, the long study duration despite a large number of centers raises doubts as to whether the study population represents the majority of patients with $\mathrm{PE}$ and right ventricular dysfunction. With this criticism in mind, it may be concluded from the trial that in very carefully selected patients with PE and right ventricular function, systemic thrombolysis can ameliorate the severity of the early course of the disease, at least in the perception of the attending physician. As in previous trials, an impact on mortality was not shown.

\section{Mechanical Thrombus Fragmentation with or without Local Thrombolysis}

Several authors have convincingly demonstrated that pulmonary artery thrombus burden can be reduced by mechanical thrombus fragmentation using catheter devices with or without local thrombolysis, thereby ensuring rapid right ventricular recovery [41-45]. There are no data as to whether this approach is more effective than systemic thrombolysis. However, it certainly does require more logistics, equipment and material. It does not appear likely that any prospective study will further investigate the potential value of this regimen. It may still be argued that mechanical thrombus fragmentation can be beneficial in hemodynamically instable patients with severe contraindications against systemic thrombolysis, such as early postoperative patients, particularly after CNS surgery or in the immediate postpartum period.

\section{Surgery}

Although a procedure with a history of more than 150 years, emergency open lung pulmonary thrombectomy should be avoided. In a remarkable prospective comparison with systemic thrombolysis, no advantage of heartlung machine-assisted thrombectomy could be found 


\begin{tabular}{ll}
\hline Clinical presentation of PE & Principles of therapy \\
\hline Cardiac arrest requiring CPR & emergency systemic thrombolysis \\
\hline $\begin{array}{l}\text { Hemodynamic shock and need for } \\
\text { catecholamines }\end{array}$ & $\begin{array}{l}\text { systemic thrombolysis, or mechanical thrombus } \\
\text { fragmentation with/without local thrombolysis if } \\
\text { available }\end{array}$ \\
\hline $\begin{array}{l}\text { Right ventricular dysfunction with } \\
\text { hemodynamic stability }\end{array}$ & $\begin{array}{l}\text { anticoagulation and intensive care; systemic throm- } \\
\text { bolysis may be considered when bleeding risk is low }\end{array}$ \\
\hline $\begin{array}{l}\text { Normal right ventricular function } \\
\text { and hemodynamic stability }\end{array}$ & $\begin{array}{l}\text { anticoagulation; outpatient treatment may be con- } \\
\text { sidered if backup facilities are available }\end{array}$ \\
\hline
\end{tabular}

[46]. It requires even more logistics than mechanical thrombus fragmentation. Theoretically, it could be an option for a patient under CPR who cannot be stabilized by systemic thrombolysis; however, to substantiate this concept, at least a couple of such cases resulting in full recovery need to be reported.

\section{Stage-Adapted Therapeutic Concept}

The presentation of patients with PE may cover a broad clinical spectrum from almost symptom-free disease to rapidly deteriorating courses or sudden death. This spectrum can be met by a range of therapeutic modalities. The prerequisite for the choice of appropriate treatment is a risk assessment which takes into account both the prognosis of the disease and the side effects of treatment. This will minimize the risk of subjecting a patient with an excellent prognosis to high-risk treatment, or of withholding procedures from patients who have almost no chance of survival unless treated vigorously. From large registries, the main short-term prognostic factors have been established to be hemodynamic parameters. Four prognostic classes may be differentiated:

(1) Cardiac arrest due to right heart failure obviously carries the highest mortality and requires CPR and intensive care. In most cases, the diagnosis can be made from the history in conjunction with bedside echocardiography. Emergency systemic thrombolysis should be performed under CPR regardless of any contraindication. Preferably, a bolus regimen of alteplase or urokinase should be administered. Major bleeding has to be expected and sufficient amounts of blood products have to be prepared.
(2) Prognosis is similarly poor for patients in shock, i.e. with a heart rate above $100 / \mathrm{min}$ and a systolic arterial pressure below $100 \mathrm{~mm} \mathrm{Hg}$. They are likely to deteriorate further within the next few hours and will likely require ventilatory support and catecholamines. Thrombolysis should be performed, e.g. $100 \mathrm{mg}$ of alteplase over $2 \mathrm{~h}$ with an initial bolus of $20 \mathrm{mg}$. If there is local expertise in mechanical thrombolysis, and it is available within $1 \mathrm{~h}$, this option may be chosen for a patient with an exceptionally high bleeding risk.

(3) Hemodynamically stable patients should be evaluated with transthoracic echocardiography in order to assess prognosis. Patients with right ventricular dysfunction have a poorer prognosis than those with normal right ventricular function. Most patients will have hypoxia and tachycardia without hypotension. They must be treated with anticoagulants and monitored in intensive care units. If right ventricular function and tachycardia do not improve within 6-12 (24) h, systemic thrombolysis may be considered with the aim of hastening recovery. Since no effect of thrombolysis on mortality has been demonstrated in these patients, contraindications against thrombolysis should be observed. Informed consent should be obtained.

(4) Hemodynamically stable patients with normal right ventricular function on echocardiography have an excellent short-term prognosis. The majority of PE patients will present in this state. Anticoagulation is mandatory, preferably with LMWHs if renal function is normal. Intensive care is not generally necessary. If transthoracic echocardiography shows normal pulmonary artery pressure (as assessed by tricuspid valve pressure gradient), even outpatient treatment can be considered, although then a stable network of backup facilities has to be available [47]. 
In summary, early assessment of the patient's shortterm prognosis is the key to a stage-adapted treatment concept. Beside clinical evaluation, echocardiography is the most important tool for risk stratification. It should be available as a bedside test in critically ill patients as well as a routine diagnostic procedure in emergency rooms. Once the diagnosis of PE has been established, echocardiography provides the basis for the principal decisions regarding both medical treatment and the setting of caregiving (table 2).

\section{References}

1 Barritt DW, Jordan SC: Anticoagulant drugs in the treatment of pulmonary embolism. A controlled trial. Lancet 1960;i:1309-1312.

2 Basu D, Gallus A, Hirsh J, Cade J: A prospective study of the value of monitoring heparin treatment with the activated partial thromboplastin time. N Engl J Med 1972;287:324327.

3 Kearon C: Drug trials that have influenced our practice in the treatment of venous thromboembolism. Thromb Haemost 1997;78:553557.

4 Bernardi E, Piccioli A, Oliboni G, Zuin R, Girolami A, Prandoni P: Nomograms for the administration of unfractionated heparin in the initial treatment of acute thromboembolism - an overview. Thromb Haemost 2000;84: 22-26.

5 Kearon C, Harrison L, Crowther M, Ginsberg JS: Optimal dosing of subcutaneous unfractionated heparin for the treatment of deep vein thrombosis. Thromb Res 2000;97:395-403.

6 Gallus A, Jackaman J, Tillett J, Mills W, Wycherley A: Safety and efficacy of warfarin started early after submassive venous thrombosis or pulmonary embolism. Lancet 1986; ii:1293-1296.

7 Brandjes DP, Heijboer H, Buller HR, de Rijk $\mathrm{M}$, Jagt $\mathrm{H}$, ten Cate JW: Acenocoumarol and heparin compared with acenocoumarol alone in the initial treatment of proximal-vein thrombosis. N Engl J Med 1992;327:14851489.

8 Low-molecular-weight heparin in the treatment of patients with venous thromboembolism. The Columbus Investigators. N Engl J Med 1997;337:657-662.

9 Simonneau G, Sors H, Charbonnier B, et al: A comparison of low-molecular-weight heparin with unfractionated heparin for acute pulmonary embolism. The THESEE Study Group. Tinzaparine ou Heparine Standard: Evaluations dans l'Embolie Pulmonaire. N Engl J Med 1997:337:663-669.

10 van Den Belt AG, Prins MH, Lensing AW, et al: Fixed dose subcutaneous low molecular weight heparins versus adjusted dose unfractionated heparin for venous thromboembolism. Cochrane Database Syst Rev 2000;(2): CD001100.
11 Huisman MV: Efficacy and safety of the oral direct thrombin inhibitor ximelagatran compared with current standard therapy for acute symptomatic deep vein thrombosis, with or without pulmonary embolism: A randomized, double-blind, multinational study. J Thromb Haemost 2003; 1(suppl 1):OC003.

12 Eriksson H, Wahlander K, Lundström T, Billing-Clason S, Schulman S, Investigators TI: Extended secondary prevention with the oral direct thrombin inhibitor ximelagatran for 18 months after 6 months of anticoagulation in patients with venous thromboembolism: A randomized, placebo-controlled trial. J Thromb Haemost 2003; 1(suppl 1):OC005.

13 The Matisse Investigators: The MATISSE-PE trial, a multicenter, randomized, open study comparing once-daily fondaparinux (Arixtra ${ }^{\circledR}$ ) with adjusted-dose intravenous unfractionated heparin (UFH) in the initial treatment of acute symptomatic pulmonary embolism (PE). J Thromb Haemost 2003; 1(suppl 1):OC331.

14 Hirsh J, Dalen J, Anderson DR, et al: Oral anticoagulants: Mechanism of action, clinical effectiveness, and optimal therapeutic range. Chest 2001;119(suppl 1):8S-21S.

15 Kearon C, Ginsberg JS, Kovacs MJ, et al: Comparison of low-intensity warfarin therapy with conventional-intensity warfarin therapy for long-term prevention of recurrent venous thromboembolism. N Engl J Med 2003;349: 631-639.

16 Crowther MA, Ginsberg JS, Julian J, et al: A comparison of two intensities of warfarin for the prevention of recurrent thrombosis in patients with the antiphospholipid antibody syndrome. N Engl J Med 2003;349:1133-1138.

17 Khamashta MA, Cuadrado MJ, Mujic F, Taub NA, Hunt BJ, Hughes GRV: The management of thrombosis in the antiphospholipid-antibody syndrome. N Engl J Med 1995;332:993997.

18 Douketis JD, Kearon C, Bates S, Duku EK, Ginsberg JS: Risk of fatal pulmonary embolism in patients with treated venous thromboembolism. JAMA 1998;279:458-462.

19 Schulman S, Rhedin AS, Lindmarker P, et al: A comparison of six weeks with six months of oral anticoagulant therapy after a first episode of venous thromboembolism. Duration of Anticoagulation Trial Study Group. N Engl J Med 1995;332:1661-1665.

20 Schulman S: Care of patients receiving longterm anticoagulant therapy. $\mathrm{N}$ Engl J Med 2003;349:675-683.
21 Hull R, Hirsh J, Jay R, et al: Different intensities of oral anticoagulant therapy in the treatment of proximal-vein thrombosis. N Engl J Med 1982;307:1676-1681.

22 Beyth RJ, Quinn LM, Landefeld S: Prospective evaluation of an index for predicting the risk of major bleeding in outpatients treated with warfarin. Am J Med 1998;105:91-99.

23 Kuijer PM, Hutten BA, Prins MH, Buller HR: Prediction of the risk of bleeding during anticoagulant treatment for venous thromboembolism. Arch Intern Med 1999;159:457-460.

24 Schulman S, Granqvist S, Holmstrom M, et al: The duration of oral anticoagulant therapy after a second episode of venous thromboembolism. The Duration of Anticoagulation Trial Study Group. N Engl J Med 1997;336:393398.

25 Kearon C, Gent M, Hirsh J, et al: A comparison of three months of anticoagulation with extended anticoagulation for a first episode of idiopathic venous thromboembolism. N Engl J Med 1999;340:901-907.

26 Agnelli G, Prandoni P, Santamaria MG, et al: Three months versus one year of oral anticoagulant therapy for idiopathic deep venous thrombosis. Warfarin Optimal Duration Italian Trial Investigators. N Engl J Med 2001; 345:165-169.

27 Ridker PM, Goldhaber SZ, Danielson E, et al: Long-term, low-intensity warfarin therapy for the prevention of recurrent venous thromboembolism. N Engl J Med 2003;348:14251434.

28 Seligsohn U, Lubetsky A: Genetic susceptibility to venous thrombosis. N Engl J Med 2001; 344:1222-1231.

29 Baglin T, Luddington R, Brown K, Baglin C: Incidence of recurrent venous thromboembolism in relation to clinical and thrombophilic risk factors: Prospective cohort study. Lancet 2003;362:523-526.

30 van der Heijden JF, Hutten BA, Büller HR, Prins MH: Vitamin K antagonists or low-molecular-weight heparin for the long term treatment of symptomatic venous thromboembolism. Cochrane Database Syst Rev 2002;(1): CD002001.

31 Agnelli G, Prandoni P, Becattini C, et al: Extended oral anticoagulant therapy after a first episode of pulmonary embolism. Ann Intern Med 2003;139:19-25.

32 Koopman MM, Buller HR: Short- and longacting synthetic pentasaccharides. $\mathrm{J}$ Intern Med 2003;254:335-342. 
33 Dalen JE, Alpert JS, Hirsch J: Thrombolytic therapy for pulmonary embolism: Is it effective? Is it safe? When is it indicated? Arch Intern Med 1997;157:2550-2556.

34 Kasper W, Konstantinides S, Geibel A, et al: Management strategies and determinants of outcome in acute major pulmonary embolism: Results of a multicenter registry. J Am Coll Cardiol 1997;30:1165-1171.

35 Arcasoy SM, Vachani A: Local and systemic thrombolytic therapy for acute venous thromboembolism. Clin Chest Med 2003;24:73-91.

36 Wood KE: Major pulmonary embolism: Review of a pathophysiologic approach to the golden hour of hemodynamically significant pulmonary embolism. Chest 2002;121:877905.

37 Kasper W, Konstantinides S, Geibel A, Tiede $\mathrm{N}$, Krause T, Just H: Prognostic significance of right ventricular afterload stress detected by echocardiography in patients with clinically suspected pulmonary embolism. Heart 1997 ; 77:346-349.
38 Kasper W, Geibel A, Tiede N, et al: Distinguishing between acute and subacute massive pulmonary embolism by conventional and Doppler echocardiography. Br Heart J 1993; 70:352-356.

39 Goldhaber SZ, Visani L, De Rosa M: Acute pulmonary embolism: Clinical outcomes in the International Cooperative Pulmonary Embolism Registry (ICOPER). Lancet 1999;353: 1386-1389.

40 Konstantinides S, Geibel A, Heusel G, Heinrich $\mathrm{F}$, Kasper W, the Management Strategies and Prognosis of Pulmonary Embolism-3 Trial Investigators: Heparin plus alteplase compared with heparin alone in patients with submassive pulmonary embolism. N Engl J Med 2002;347: 1143-1150.

41 Reekers JA, Baarslag HJ, Koolen MG, Van Delden O, Van Beek EJ: Mechanical thrombectomy for early treatment of massive pulmonary embolism. Cardiovasc Intervent Radiol 2003; 26:246-250.

42 Muller-Hulsbeck S, Brossmann J, Jahnke T, et al: Mechanical thrombectomy of major and massive pulmonary embolism with use of the Amplatz thrombectomy device. Invest Radiol 2001;36:317-322.
43 Fava M, Loyola S, Huete I: Massive pulmonary embolism: Treatment with the hydrolyser thrombectomy catheter. J Vasc Interv Radiol 2000;11:1159-1164.

44 Schmitz-Rode T, Janssens U, Duda SH, Erley CM, Gunther RW: Massive pulmonary embolism: Percutaneous emergency treatment by pigtail rotation catheter. $\mathrm{J}$ Am Coll Cardiol 2000;36:375-380.

45 Murphy JM, Mulvihill N, Mulcahy D, Foley B, Smiddy P, Molloy MP: Percutaneous catheter and guidewire fragmentation with local administration of recombinant tissue plasminogen activator as a treatment for massive pulmonary embolism. Eur Radiol 1999;9:959-964.

46 Gulba DC, Schmid C, Borst HG, Lichtlen P, Dietz R, Luft FC: Medical compared with surgical treatment for massive pulmonary embolism. Lancet 1994;343:576-577.

47 Beer JH, Burger M, Gretener S, Bernard-Bagattini $\mathrm{S}$, Bounameaux $\mathrm{H}$ : Outpatient treatment of pulmonary embolism is feasible and safe in a substantial proportion of patients. J Thromb Haemost 2003; 1:186-187. 